Article

\title{
Increasing Resolution in Live Cell Microscopy by Structured Illumination (SIM)
}

\author{
Verena Richter, Mathis Piper, Michael Wagner and Herbert Schneckenburger * \\ Institute of Applied Research, Aalen University, Beethovenstr. 1, 73430 Aalen, Germany; \\ verena.richter@hs-aalen.de (V.R.); mathis.piper@t-online.de (M.P.); michael.wagner@hs-aalen.de (M.W.) \\ * Correspondence: Herbert.Schneckenburger@hs-aalen.de; Tel.: +49-7361-5763401
}

Received: 31 January 2019; Accepted: 15 March 2019; Published: 20 March 2019

Featured Application: Super-resolution microscopy of living cells and tissues.

\begin{abstract}
In the context of various approaches to super-resolution microscopy, structured illumination microscopy (SIM) offers several advantages: it needs rather low light doses (with a low risk of phototoxicity or photobleaching), is comparably fast and flexible concerning the use of microscopes, objective lenses and cameras, and has potential for 3D imaging. This paper describes an experimental setup for SIM with first diffraction orders of a spectral light modulator (SLM) creating an interference pattern in two dimensions. We kept this system rather compact with a comparably large illuminated object field, validated it with nano-beads and applied it further to living cells for imaging the cytoskeleton, mitochondria or cell nuclei with a resolution slightly above $100 \mathrm{~nm}$. Its advantages, challenges and limitations - concerning cameras, acquisition time, depth of imaging, light exposure, and combining it with further super-resolving methods-are discussed.
\end{abstract}

Keywords: live cell imaging; super-resolution microscopy; SIM; deep view imaging; nano-beads; cytoskeleton; mitochondria; doxorubicin

\section{Introduction}

Resolution in microscopy is well described by the Abbe or the Rayleigh criterion. In the first case, resolution is determined by diffraction of a coherent light beam and results in $y_{\min } \geq \lambda / 2 \mathrm{~A}_{N}$ (with $\lambda$ corresponding to the wavelength of light and $A_{N}$ to the numeric aperture of the microscope objective lens), whereas in the second case, $y_{\min }=0.61 \lambda / A_{N}$ results from the diffraction function of an incoherently luminescent spot (Airy disk). In both cases, values around $200 \mathrm{~nm}$ are attained for high numeric apertures $\left(A_{N} \geq 1.30\right)$ and around $400-500 \mathrm{~nm}$ for moderate apertures $\left(A_{N} \leq 0.60\right)$, e.g., upon imaging of larger objects by long distance objective lenses.

Optical microscopy provides a depth of focus $\mathrm{L}=\mathrm{n} \lambda / \mathrm{A}_{\mathrm{N}}{ }^{2}$, which is in the range of about $400 \mathrm{~nm}$ up to a few micrometers. This implies that only from thin object layers can sharp images be generated, and that further techniques are needed for imaging 3D samples. Three dimensional resolution has been introduced by confocal laser scanning microscopy (CLSM), where the focus of a laser beam scanning the sample is imaged on a pinhole in front of the detector, while all of the out-of-focus information is suppressed [1,2]. By moving the sample step-by-step in a vertical direction, images of numerous planes can thus be recorded and combined in a 3D image. Alternatively, samples are illuminated by a thin light sheet that is in the focus of the detection lens (light-sheet fluorescence microscopy, LSFM). Such light sheets are generated either by a cylindrical lens or by laser beam scanning [3,4]. The 3D image is acquired by either moving the light sheet in the vertical direction with a simultaneous shift of the detection lens, or by moving the sample through the image plane. In contrast to CLSM only the plane of detection has to be exposed to light, so that the total light exposure in 3D imaging is considerably 
lower. This favors LSFM for experiments performed over a long time period. Experimental setups for LSFM range from stand-alone microscopes to miniaturized modules, which can be adapted to various kinds of commercial microscopes and combined with further experimental techniques [5].

Only in the last 25 years have methods been described with a resolution below the Abbe criterion. They are summarized under the term "super-resolution microscopy", and include:

- $\quad$ stimulated emission depletion (STED) microscopy [6], where the outer regions of an illuminated spot are depleted by stimulated emission within the focus of a second donut-shaped laser beam upon optical excitation in a laser scanning microscope. While a fluorescent spot may thus be confined to $70 \mathrm{~nm}$ or less, the irradiance exceeds that of a conventional fluorescence microscope by a factor of $10^{4}-10^{5}$, so at the moment live-cell imaging does not appear to be possible, although various approaches to reduce the light exposure considerably are being pursued. Those methods include reduction of state transition cycles (REScue) [7], adaptive-illumination STED nanoscopy [8] or MINFLUX technology with tracking of single molecules in the center of a donut-shaped laser beam [9];

- $\quad$ super-localization microscopy of single molecules located within a thin illuminated layer of a sample. If a single molecule is detected $\mathrm{n}$ times, its localization can be determined with precision, $\Delta \mathrm{x}=\Delta \mathrm{x}_{0} / \sqrt{ } \mathrm{n}$ with $\Delta \mathrm{x}_{0} \approx 200 \mathrm{~nm}$ resulting from the Rayleigh criterion. Therefore, a precision of localization $\Delta \mathrm{x}=20 \mathrm{~nm}$ results when $\mathrm{n}=100$ and $\Delta \mathrm{x}=10 \mathrm{~nm}$ when $\mathrm{n}=400$. Methods based on super-localization microscopy include stochastic optical reconstruction microscopy (STORM), photoactivation localization microscopy (PALM) and related techniques [10-12]. However, the irradiance needed for single-molecule experiments is still 500-1000 times higher than in conventional fluorescence microscopy, such that cells are expected to survive only a short time.

Furthermore, the resolution in CLSM can be increased up to a factor of two if only part of an Airy disk passes the detection pinhole, or if each Airy disk is detected by a large number of "infinitely small pinholes". This becomes possible by using several single-detector elements (Airy scan microscopy) or an appropriate camera (image scanning microscopy [13]) as well as by scanning the surface of the pinhole (rescanning confocal microscopy, RCM) [14].

In addition to various scanning techniques, as well as to single-molecule localization techniques, structured illumination microscopy (SIM) has proven its super-resolution potential $[15,16]$. A down-modulation of conventionally non-transferable higher spatial frequencies by a periodically-modulated illumination pattern leads to resolution enhancement up to a factor of two compared to the value given by the Abbe criterion. While illumination with three laser beams creates an interference pattern with high spatial resolution in all three dimensions [17], an interference pattern in two dimensions is generated by illumination with two laser beams, e.g., the first diffraction orders of an optical grating or a spatial light modulator (SLM) [18]. Calculation of a super-resolved image commonly needs 9-15 single images with structured illumination of different phases and rotation angles $[17,18]$. This can be achieved by the fast scanning of a diffraction element with galvano mirrors, as described in [19] and further realized in a commercial system (Elyra 7, Carl Zeiss Jena, Germany). Use of a switchable SLM, however, avoids any movable component and may improve the stability of the whole system. However, low diffraction angles of the first interference order require long optical path lengths for creating an appropriate interference pattern, and furthermore result in small object fields within the microscope. In comparison with the SLM-based system described in [18] it was, therefore, our aim to develop a more compact setup with a larger illuminated object field. Furthermore, this manuscript describes several applications in live cell microscopy and addresses some of the principal requirements. Light exposure in SIM exceeds that of conventional wide-field microscopy only slightly, and is considerably lower than exposures needed for STED, PALM or STORM ([20] and references therein), as well as Airy scan microscopy. This favors SIM for live-cell microscopy, especially if longer exposure times or repeated measurements are required. 


\section{Materials and Methods}

Fluorescent polystyrene beads of $100 \mathrm{~nm}$ and $200 \mathrm{~nm}$ diameter were kindly provided by Florian Schock (Institute of Pharmacy and Molecular Biotechnology, University of Heidelberg, Germany), and calibration slides were prepared as described in Reference [21]. 3T3 murine fibroblasts were cultivated as monolayers or multilayers on microscope cover slips (without any further substrates) in Dulbecco's modified Eagle medium (DMEM) supplemented with 10\% fetal calf serum, pyruvate and antibiotics at $37^{\circ} \mathrm{C}$ and $5 \% \mathrm{CO}_{2} .72 \mathrm{~h}$ after seeding cells were incubated for $20 \mathrm{~min}$ with Tubulin Tracker ${ }^{\mathrm{TM}}$ Green at a concentration of $250 \mathrm{nM}$. MCF-7 human breast cancer cells were cultivated as monolayers on microscope cover slips in supplemented Dulbecco's MEM/Ham F-12 at $37{ }^{\circ} \mathrm{C}$ and $5 \% \mathrm{CO}_{2}$. Cells were incubated with the cytostatic agent doxorubicin $(4 \mu \mathrm{M})$ for $24 \mathrm{~h}$ or with the mitochondrial marker rhodamine $123(5 \mu \mathrm{M})$ for $30 \mathrm{~min}$. In all cases cells were rinsed with Earle's Balanced Salt Solution (EBSS) prior to fluorescence microscopy. An argon ion laser with $\lambda_{0}=488 \mathrm{~nm}$ was used for excitation, and fluorescence was detected in an inverted microscope (Axiovert 200M, Carl Zeiss Jena, Germany) using a Plan-Neofluar $40 \times / 1.30$ (oil immersion) objective lens, a long pass filter for $\lambda_{\mathrm{D}} \geq 515 \mathrm{~nm}$ and further optics as described below.

As reported earlier [17], SIM in 2 dimensions needs an interference pattern recorded for a minimum of three phases $(0,2 \pi / 3,4 \pi / 3)$ at three rotation angles (e.g., $0^{\circ}, 60^{\circ}$ and $\left.120^{\circ}\right)$ in order to obtain an optical transfer function (OTF) of rotational symmetry (Figure 1). Interference patterns of an SLM (ferroelectric LCOS, SXGA-3DM, Forth Dimension Displays, UK) are created by a computer program, and first orders of diffraction of an argon ion laser beam $\left(\lambda_{0}=488 \mathrm{~nm}\right)$ are used for structured illumination, as reported in Reference [18]. Compared to conventional gratings, no movable components are needed; however, the "grating constant" of the SLM of about $90 \mu \mathrm{m}$ results in low diffraction angles and requires a large focal length on the collecting lens $\mathrm{L}_{1}$ (Figure 2) for geometrical separation of the diffraction orders. The SLM is imaged by a telescope system consisting of the lenses $\mathrm{L}_{1}$ (focal length $\mathrm{f}_{1^{\prime}}=500 \mathrm{~mm}$ ) and $\mathrm{L}_{2}\left(\mathrm{f}_{2^{\prime}}=120 \mathrm{~mm}\right.$ ). This gives an interference pattern of the first diffraction orders with a grating constant of $10.96 \mu \mathrm{m}$ in the image plane of the SLM, which is reduced to $\Lambda=274 \mathrm{~nm}$ by the microscope objective lens $(40 \times / 1.30$ oil immersion). The magnification factor of the objective lens includes that of the tube lens (TL) (also depicted in Figure 2). The resolution in the present case can be calculated by summing up the spatial frequencies resulting from the Abbe criterion $\mathrm{k}_{\text {Abbe }}=2 \mathrm{~A}_{\mathrm{N}} / \lambda=0.00533 \mathrm{~nm}^{-1}$ (with $\lambda=488 \mathrm{~nm}$ ) and the interference pattern $\mathrm{k}_{\text {grating }}=$ $1 / \Lambda=0.00365 \mathrm{~nm}^{-1}$ as $\mathrm{d}_{\min }=\left(\mathrm{k}_{\text {Abbe }}+\mathrm{k}_{\text {grating }}\right)^{-1}=111 \mathrm{~nm}$. In comparison with the Abbe criterion $\lambda / 2 \mathrm{~A}_{\mathrm{N}}=188 \mathrm{~nm}$ this is an improvement by a factor of 1.70 . Further components needed for structured illumination (Figure 2) are a $\lambda / 4$ plate, a plate of 6 pinholes (to select the first diffraction order of the SLM for $0^{\circ}, 60^{\circ}$ and $120^{\circ}$ orientation) and a so-called "pizza polarizer" to select an azimuthal polarization in each case. In addition, an aperture of 5-8 $\mathrm{mm}$ on the microscope objective lens is required for transmission of the two interfering laser beams. The illuminated object field of the sample results from diminution of the expanded laser beam (original diameter on the SLM was about $10 \mathrm{~mm}$ ) by the telescope consisting of the lenses $\mathrm{L}_{1}$ and $\mathrm{L}_{2}$, as well as by the microscope objective lens, and amounts to about $60 \mu \mathrm{m}$. Fluorescence images are recorded either with a ProgRes C10 CCD camera (Jenoptik GmbH, Jena, Germany) or an Orca Flash camera set C11440-10C (Hamamatsu Photonics Deutschland $\mathrm{GmbH}$, Herrsching, Germany) with a pixel size of $3.45 \mu \mathrm{m}$ or $3.63 \mu \mathrm{m}$, respectively. Therefore, objects down to a size of about $100 \mathrm{~nm}$ are resolved by a $40 \times$ microscope objective lens. In addition, the Orca camera can be synchronized with the SLM, so that the camera integrates the images when the SLM displays the relevant pattern, and the switching time of a few milliseconds becomes negligible compared to the usual exposure time of $0.5 \mathrm{~s}$. After the acquisition of 9 structured images (within about $5 \mathrm{~s}$ ) a super-resolution image is calculated using Fourier transformation [22]. 


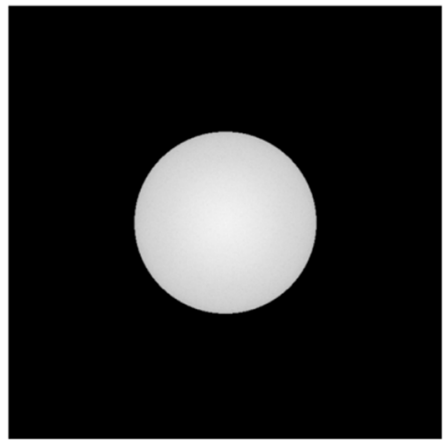

a

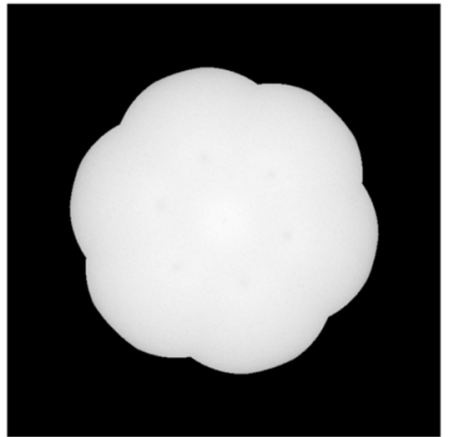

b

Figure 1. Comparison of optical transfer functions (OTFs): Wiener filtered wide-field (a) and structured illumination with two interfering laser beams at angles of $0^{\circ}, 60^{\circ}$ and $120^{\circ}(\mathbf{b})$.

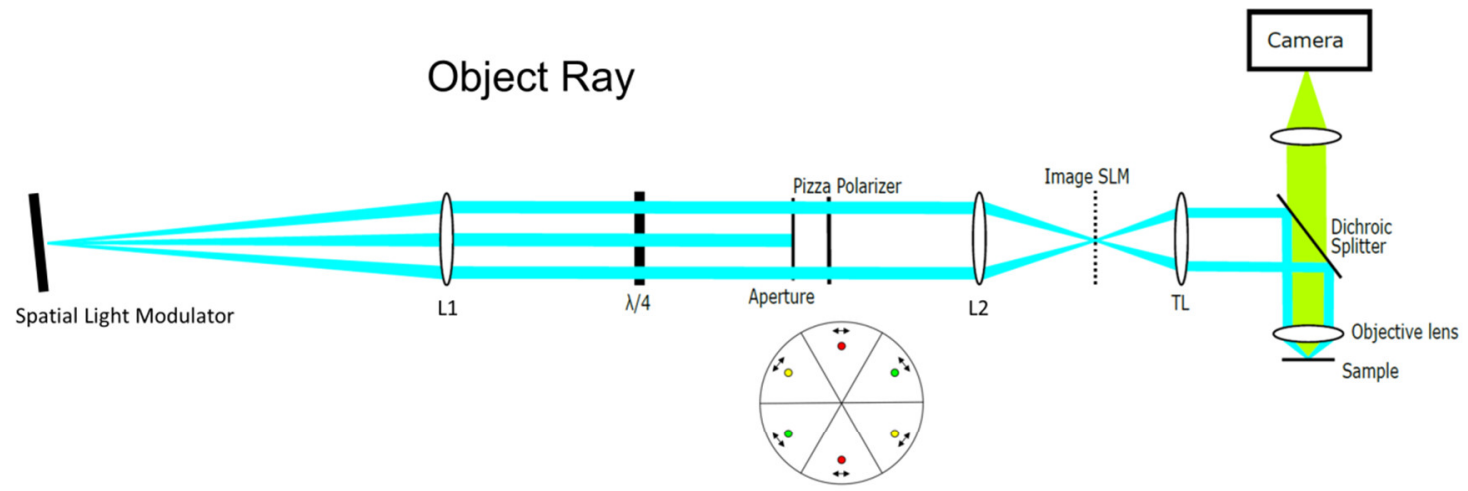

Illumination Ray

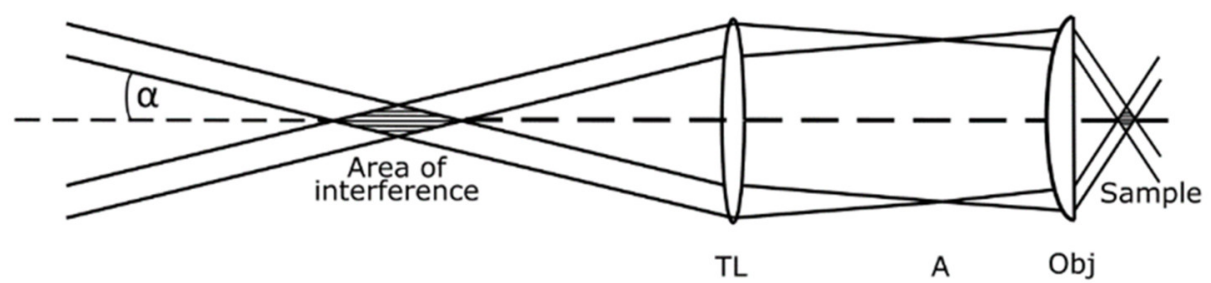

Figure 2. Experimental setup for SIM: The object ray creates an image of the SLM pattern in the plane of the sample upon illumination by the parallel beam of an argon ion laser $\left(\lambda_{0}=488 \mathrm{~nm}\right.$ expanded by a telescope to a diameter of $10 \mathrm{~mm}$, not shown); the illumination ray is focused in the aperture plane A of the objective lens and results in two parallel beams interfering in the plane of the sample (TL = tube lens, Obj = microscope objective lens). The inset shows a scheme of the "pizza polarizer" with 6 areas of different polarization.

\section{Results}

Fluorescence images of the polystyrene nano-beads (diameters of $100 \mathrm{~nm}$ and $200 \mathrm{~nm}$ ) are depicted in Figure 3 as conventional wide-field images $(a, d)$, wide-field images with a Wiener filter for noise suppression (b,e), and SIM images (c,f). In Figure $3 a, b$ resolution according to the Abbe criterion is lower, and in Figure 3d,e close to the particle size. In addition, particles appear somehow blurred in Figure 3a,d, and overlapping in Figure $3 a, b, d, e$, while in Figure 3c,f all particles appear clear and well resolved. 


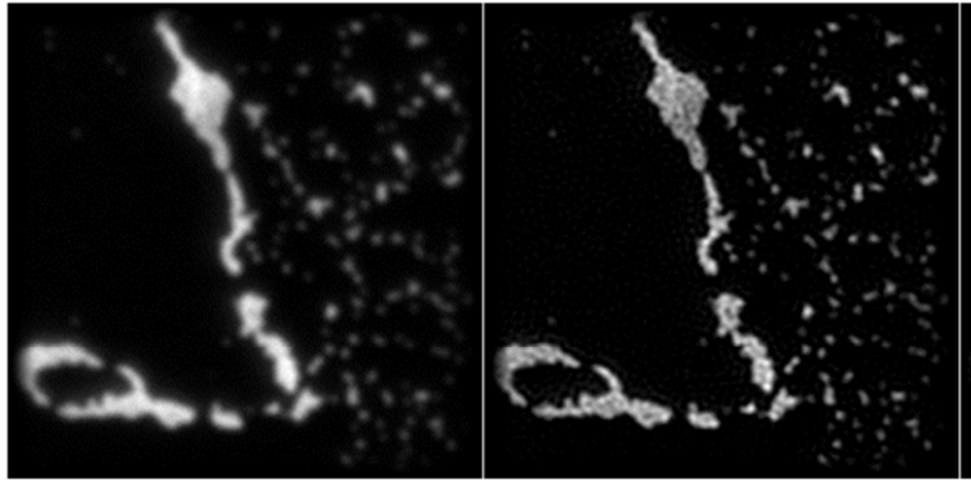

a

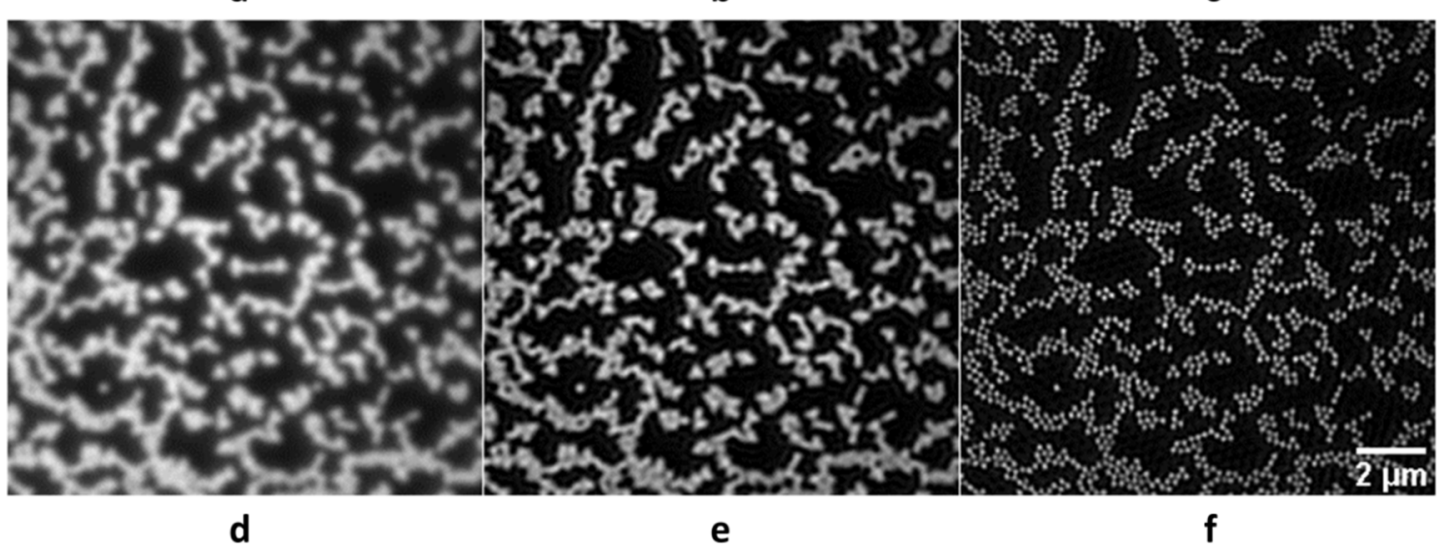

Figure 3. Images of fluorescent nano-beads with a size of $100 \mathrm{~nm}$ (upper part) as well as $200 \mathrm{~nm}$ (lower part) using wide-field microscopy $(\mathbf{a}, \mathbf{d})$, wide-field microscopy with a Wiener filter (b,e) or SIM (c,f); excitation wavelength: $\lambda_{0}=488 \mathrm{~nm}$; detection range: $\lambda_{\mathrm{D}} \geq 515 \mathrm{~nm}$; Plan-Neofluar $40 \times / 1.30$ oil immersion objective lens.

In Figure 4a a fluorescence image of a 3T3 fibroblast incubated with Tubulin Tracker ${ }^{\mathrm{TM}}$ Green ( $250 \mathrm{nM}$ for $20 \mathrm{~min}$ ) is depicted, and the image shows brightly fluorescent microtubules. In comparison with the wide-field image the SIM image (b) shows an improved resolution, and in combination with the software algorithm described in Reference [22] permits detection of a single cell layer. The improvement of resolution by a factor of about 1.7 is also documented by a line scan with Gaussian fit over $0.8 \mu \mathrm{m}$ (c). Here, the calculated diameters of $190 \mathrm{~nm}$ (wide-field microscopy with Wiener filter) as well as $115 \mathrm{~nm}$ (SIM) fit the theoretical values of $187 \mathrm{~nm}$ and $111 \mathrm{~nm}$, respectively, and confirm previous results obtained with other tubulin markers $[23,24]$. It should be mentioned that the real diameter of a microtubule is about $30 \mathrm{~nm}$ and, therefore, considerably smaller.

A further application is presented in Figure 5 for doxorubicin (adriamycin), an anthracycline antibiotic, which has been used as a cytostatic drug in cancer chemotherapy for several decades [25]. The drug is taken up by cells due to passive diffusion through the membrane and finally intercalates in DNA strands, where it causes chromatin condensation and initiates apoptosis [26]. Location in the cell nuclei of MCF-7 breast cancer cells is well documented in Figure 5, but in comparison with wide-field microscopy (b), SIM gives an improved resolution for the nuclear envelope from about $220 \mathrm{~nm}$ to $120-150 \mathrm{~nm}$ (c) and possibly some information on nuclear architecture upon application of this cytostatic agent. 


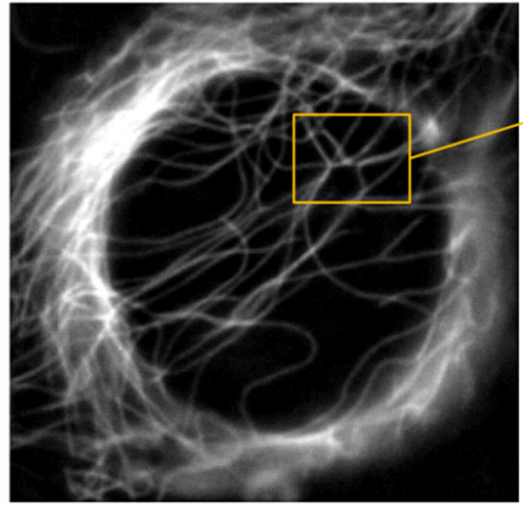

a
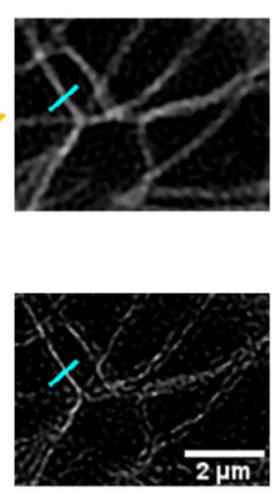

b

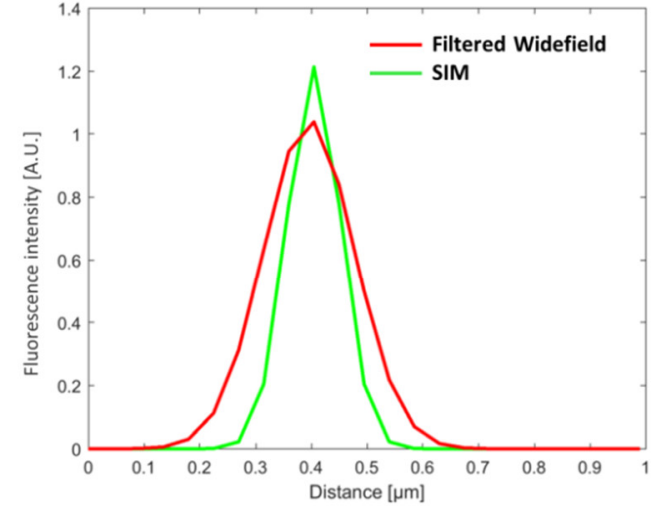

C

Figure 4. Fluorescence image of a $3 \mathrm{~T} 3$ fibroblast incubated with Tubulin Tracker ${ }^{\mathrm{TM}}$ Green (250 nM for $20 \mathrm{~min}$ ): (a) wide-field microscopy with Wiener filter, (b) comparison of filtered wide-field image and SIM for a selected field, and (c) line scan with Gaussian fit over a marked range of $0.8 \mu \mathrm{m}$ for filtered wide-field and SIM ("full result"). Excitation wavelength: $\lambda_{0}=488 \mathrm{~nm}$; detection range: $\lambda_{\mathrm{D}} \geq 515 \mathrm{~nm}$; Plan Neofluar $40 \times / 1.30$ oil immersion objective lens.

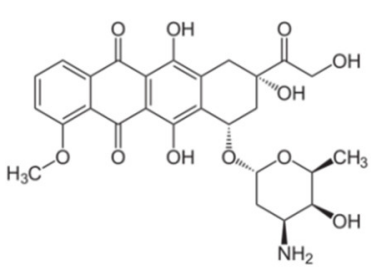

a

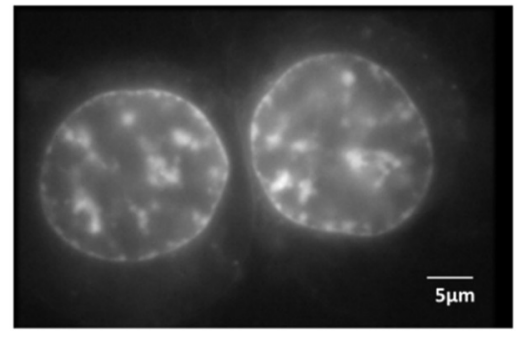

b

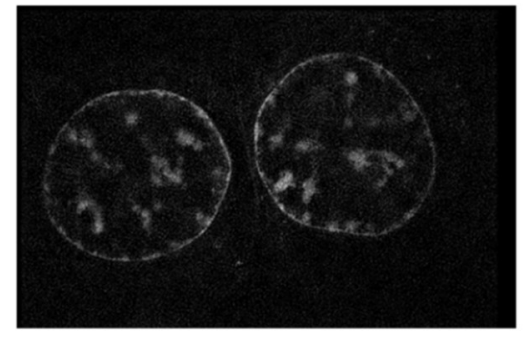

C

Figure 5. Molecular structure of doxorubicin (a); fluorescence images of MCF-7 breast cancer cells incubated with doxorubicin $(4 \mu \mathrm{M}, 24 \mathrm{~h})$ using wide-field microscopy (b), SIM (c); excitation wavelength: $\lambda_{0}=488 \mathrm{~nm}$; detection range: $\lambda_{\mathrm{D}} \geq 515 \mathrm{~nm}$; Plan Neofluar $40 \times / 1.30$ oil immersion objective lens.

Fluorescence images of individual MCF-7 breast cancer cells incubated with the mitochondrial marker rhodamine 123 (5 $\mathrm{MM}$ for $30 \mathrm{~min}$ ) are depicted in Figure 6. In both parts of this image a non-fluorescent nucleus is surrounded by mitochondria which appear as long fluorescent rods. While the wide-field image (Figure 6a) shows some overlaying, diffuse, and out-of-focus fluorescence, the image calculated from nine images with structured illumination (SIM shown in Figure 6b) shows fluorescence only from the focal plane with enhanced image quality. However, the resolution cannot be specified, since the mitochondria are larger than the resolution in the microscope, and mitochondrial sub-structures cannot be visualized. However, this kind of experiment may be useful to measure the mitochondrial membrane potential [27] and possible malfunctions of the respiratory chain [28].

In contrast to the cell monolayers depicted in Figures 4-6, fluorescence images of multilayers appear blurred with large amounts of scattering and out-of-focus fluorescence. If the focus is adjusted at a sample depth of 10-20 $\mu \mathrm{m}$, evaluation of a SIM image is still possible; however, sub-cellular structures are less resolved, as demonstrated in Figure 7. The possibilities for image improvement are discussed below. 


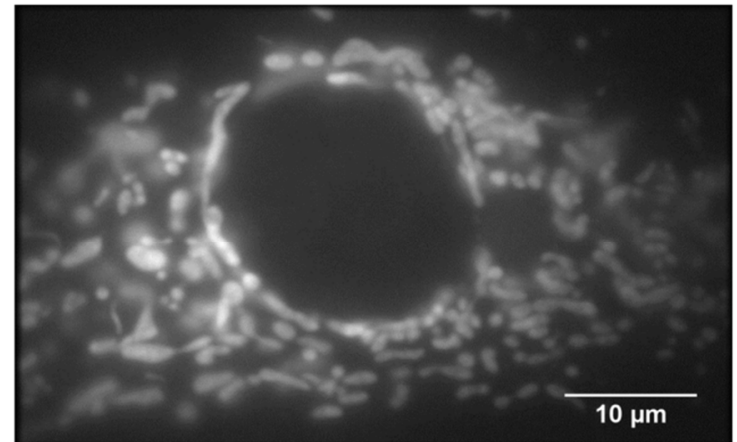

a

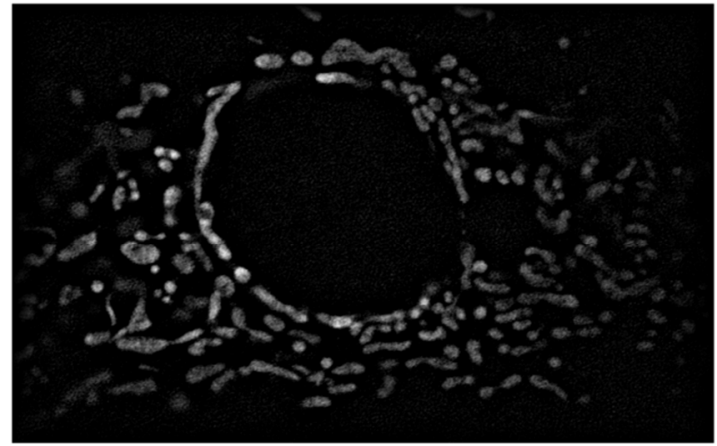

b

Figure 6. MCF-7 breast cancer cells incubated with the mitochondrial marker R123 ( $5 \mu \mathrm{M}, 30$ min) wide field image (a) and SIM image (b) (evaluated by R. Heintzmann, IPHT Jena); excitation wavelength: $\lambda_{0}$ $=488 \mathrm{~nm}$; detection range: $\lambda_{\mathrm{D}} \geq 515 \mathrm{~nm}$; Plan Neofluar $40 \times / 1.30$ oil immersion objective lens.

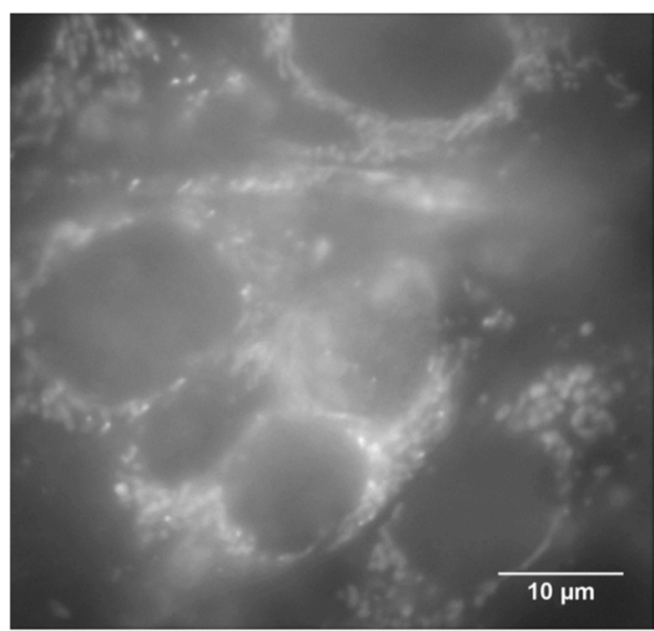

a

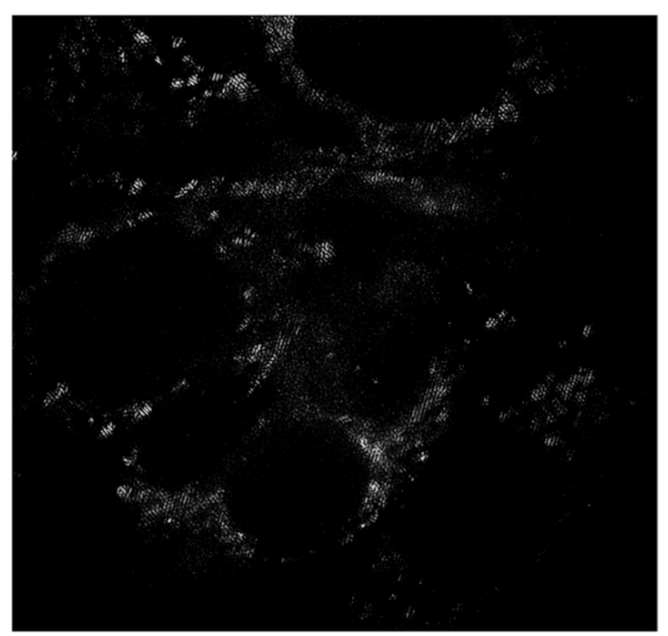

b

Figure 7. 3T3 fibroblasts from a multilayer incubated with the mitochondrial marker R123 (5 $\mu \mathrm{M}$ for $30 \mathrm{~min}$ ); wide field image (a) and SIM image (b) in a sample depth $\Delta \mathrm{z}=10 \mu \mathrm{m}$. Excitation wavelength: $\lambda_{0}=488 \mathrm{~nm}$; detection range: $\lambda_{\mathrm{D}} \geq 515 \mathrm{~nm}$; Plan Neofluar $40 \times / 1.30$ oil immersion objective lens.

\section{Discussion}

Increasing the resolution in comparison with the Abbe or Rayleigh criterion is still a challenge in optical microscopy. As reported above, SIM is a promising approach in view of the low light exposure needed for imaging living cells. In the present manuscript an increase of the resolution by a factor of 1.7 (theoretically up to a factor of two) has been well documented for two-dimensional samples, e.g., fluorescent beads or cell monolayers. The gain in resolution is still low in comparison to STED or super-localization microscopy, e.g., STORM, where growth of protein fibrils was recorded with a resolution better than $20 \mathrm{~nm}$ [29]. However, those methods require increased light exposure and often are not compatible with live cell microscopy.

Microscopy of three-dimensional specimens, e.g., cell multilayers, spheroids or tissue biopsies, is still a challenge for all kinds of super-resolution microscopy. In the case of SIM an increase of resolution up to a factor of two can be theoretically maintained in larger depths of a sample, even if long distance objective lenses of lower numeric aperture are needed. However, images are subject to pronounced light scattering: they appear blurred (see Figure 7), and low contrast interference patterns, e.g., those of a spatial light modulator, are hardly visible. This makes the calculation of high-resolution SIM images very difficult. However, it appears possible to combine structured illumination and light sheet microscopy [5] (in an up-modulation mode) and to suppress scattered light outside the light 
sheet. In this way, imaging in larger depths of the sample may become possible. Furthermore, precise positioning of the specimen in the axial direction is cogent for imaging $3 \mathrm{D}$ samples. This option is not available in many customary microscopes, where only the objective lens, but not the specimen can be moved in the axial direction. Therefore, with a fixed position for the tube lens (Figure 2) the interference pattern of two overlapping beams is only maintained over a small depth of the specimen. However, coupling of the sample to a piezo element on the microscope stage permits moving of the sample in the axial direction and seems to solve this problem.

The main characteristics of the present SIM system can be summarized as follows:

- $\quad$ it is a wide-field method requiring only low light doses (up to about $10 \mathrm{~J} / \mathrm{cm}^{2}$ ) which permits keeping cells alive after incubation with a fluorescence marker or transfection with a fluorescent protein [20] and also avoids artefacts due to photobleaching [30];

- $\quad$ it is comparably simple, without any moving components, using a commercially available (inverted) microscope;

- microscope lenses of various magnification and aperture can be used. In the present case a $40 \times / 1.30$ objective lens permits obtaining a comparably large field of illumination for cell-based experiments, but requires cameras with more than 250 pixels per millimeter (e.g., ProgRes cameras by Jenoptik GmbH or an Orca Flash camera set by Hamamatsu Photonics) to image structures of about $100 \mathrm{~nm}$ size; and,

- $\quad$ it has the potential for imaging in larger depths of a sample ("deep view imaging"). This potential can, in principle, even be used for in combination with further super-resolution methods, e.g., STED microscopy where SIM may generate a three-dimensional depletion pattern, permitting axial super-resolution STED with moderate light exposure [31].

Although each SIM image has to be calculated from at least nine individual images, acquisition times are short compared to most other super-resolution techniques based on laser beam scanning or on single-molecule localization (only for LSM 880 with Airy scan, Carl Zeiss Jena, Germany, recording times down to about $50 \mathrm{~ms}$ are reported). Our present acquisition time for one super-resolution image (including nine single images) is about $5 \mathrm{~s}$, but this time can be shortened to the millisecond range [30,32] using either galvano scanners (e.g., Elyra 7, Carl Zeiss Jena, Germany) or a spectral light modulator (SLM). So far, the highest frame rate (255 frames per second) was achieved with Elyra 7 and lattice SIM, a technique creating a three-dimensional point pattern (instead of a linear grid) operated in the so-called "burst" mode. This technique [33] has the advantage that for recording individual images the pattern has only to be shifted (not rotated), so the image sequence becomes faster. In addition, the modulation depth of lattice SIM is enhanced in comparison with conventional SIM. Compared to scanning techniques, the SLM does not need any movable components, but when selecting the first orders of diffraction, many higher orders of interference have to be discarded. Therefore, either higher laser powers or longer recording times as compared to those of the scanning SIM are required. However, in all cases super-resolution images must be calculated offline from individual SIM images. It should be emphasized that in comparison to commercial systems, our present SIM setup is not optimized for the highest speed. However, it has the advantage that it uses a conventional inverted microscope and can be easily combined with other microscopy techniques, e.g., light sheet microscopy [5], as well as spectral imaging or fluorescence lifetime imaging microscopy (FLIM).

In summary, low recording times in combination with low light exposure and the potential for 3D or deep view microscopy might favor SIM in comparison with other super-resolution methods, and SIM might represent a less light consuming alternative for STED or super-localization microscopy in long-term imaging of cell or tissue dynamics [34,35]. For an increase in axial resolution close to surfaces (e.g., cell membranes), SIM can be combined with total internal reflection microscopy (TIRFM) if an interference structure is created by two laser beams whose angles of incidence are above the critical angle of total internal reflection [36]. Finally, some indirect methods, e.g., Förster resonance 
energy transfer [37], may further improve the potential of measuring molecular distances in the nanometer range.

Author Contributions: SIM experiments and evaluation, V.R.; setting up of equipment, M.P.; technical support, M.W.; organization and writing of this manuscript as well as presentation at LALS 2018, H.S.

Funding: This project was funded by the Ministry of Science and Arts (MWK) Baden-Württemberg.

Acknowledgments: The authors thank Rainer Heintzmann and Ronny Förster (IPHT Jena) as well as Christoph Cremer and Florian Schock (Univ. Heidelberg) for their valuable cooperation and Claudia Hintze (Aalen University) for skillful technical assistance.

Conflicts of Interest: The authors declare no conflict of interest.

\section{References}

1. Pawley, J. Handbook of Biological Confocal Microscopy, 3rd ed.; Springer: Boston, MA, USA, 1990. [CrossRef]

2. Webb, R.H. Confocal optical microscopy. Rep. Prog. Phys. 1996, 59, 427-471. [CrossRef]

3. Pampaloni, F.; Chang, B.-J.; Stelzer, E.H.K. Light sheet-based fluorescence microscopy (LSFM) for the quantitative imaging of cells and tissue. Cell Tissue Res. 2015, 362, 265-277. [CrossRef]

4. Santi, P.A. Light sheet fluorescence microscopy: A review. J. Histochem. Cytochem. 2011, 59, 129-138. [CrossRef] [PubMed]

5. Bruns, T.; Bauer, M.; Bruns, S.; Meyer, H.; Kubin, D.; Schneckenburger, H. Miniaturized modules for light sheet microscopy with low chromatic aberration. J. Microsc. 2016, 264, 261-267. [CrossRef]

6. Hell, S.W.; Wichmann, J. Breaking the diffraction resolution limit by stimulated emission: Stimulatedemission-depletion fluorescence microscopy. Opt. Lett. 1994, 19, 780-782. [CrossRef]

7. Staudt, T.; Engler, A.; Rittweger, E.; Harke, B.; Engelhardt, J.; Hell, S.W. Far-field optical nanoscopy with reduced number of state transition cycles (REScue). Opt. Express 2011, 19, 5644-5657. [CrossRef] [PubMed]

8. Heine, J.; Reuss, M.; Harke, B.; D’Este, E.; Sahl, S.J.; Hell, S.W. Adaptive illumination STED nanoscopy. Proc. Natl. Acad. Sci. USA 2017, 114, 9797-9802. [CrossRef]

9. Balzarotti, F.; Eilers1, Y.; Gwosch, K.C.; Gynnå, A.H.; Westphal, V.; Stefani, F.D.; Elf, J.; Hell, S.W. Nanometer resolution imaging and tracking of fluorescent molecules with minimal photon fluxes. Science 2017, 345, 606-612. [CrossRef]

10. Betzig, E.; Patterson, G.H.; Sougrat, R.; Lindwasser, O.W.; Olenych, S.; Bonifacino, J.S.; Davidson, M.W.; Lippincott-Schwartz, J.; Hess, H.F. Imaging intracellular fluorescent proteins at nanometer resolution. Science 2006, 313, 1642-1645. [CrossRef]

11. Rust, M.J.; Bates, M.; Zhuang, X. Sub-diffraction-limit imaging by stochastic optical reconstruction microscopy (STORM). Nat. Methods 2006, 3, 793-796. [CrossRef]

12. Cremer, C.; Masters, B.R. Resolution enhancement techniques in microscopy. Eur. Phys. J. H 2013, 38, 281-344. [CrossRef]

13. Müller, C.B.; Enderlein, J. Image scanning microscopy. Phys. Rev. Lett. 2010, 104, 198101. [CrossRef]

14. de Luca, G.M.R.; Breedijk, R.M.P.; Brandt, R.A.J.; Zeelenberg, C.H.C.; de Jong, B.E.; Timmermans, W.; Nahidi Azar, L.; Hoebe, R.A.; Stallinga, S.; Manders, E.M.M. Re-scan confocal microscopy: Scanning twice for better resolution. Biomed. Opt. Express 2013, 4, 2644-2656. [CrossRef]

15. Heintzmann, R.; Cremer, C. Laterally modulated excitation microscopy: Improvement of resolution by using a diffraction grating. In Optical Biopsies and Microscopic Techniques III, Proceedings of the SPIE 3568, Stockholm, Sweden, 8-12 September 1998; SPIE: Bellingham, WA, USA, 1999; pp. 185-196. [CrossRef]

16. Gustafsson, M.G.L.; Shao, L.; Carlton, P.M.; Wang, C.J.R.; Golubovskaya, I.N.; Cande, W.Z.; Agard, D.A.; Sedat, J.W. Three-dimensional resolution doubling in wide-field fluorescence microscopy by structured illumination. Biophys. J. 2008, 94, 4957-4970. [CrossRef] [PubMed]

17. Gustafsson, M.G.L. Surpassing the lateral resolution limit by a factor of two using structured illumination microscopy. J. Microsc. 2000, 198, 82-87. [CrossRef] [PubMed]

18. Förster, R.; Lu-Walther, H.-W.; Jost, A.; Kielhorn, M.; Wicker, K.; Heintzmann, R. Simple structured illumination microscope setup with high acquisition speed by using a spatial light modulator. Opt. Express 2014, 22, 20663-20677. [CrossRef] 
19. Chen, Y.; Cao, R.; Liu, W.; Zhu, D.; Zhang, Z.; Kuang, C.; Liu, X. Widefield and total internal reflection fluorescent structured illumination microscopy with scanning galvo mirrors. J. Biomed. Opt. 2018, 23, 1-9. [CrossRef] [PubMed]

20. Schneckenburger, H.; Richter, V.; Wagner, M. Live-Cell Optical Microscopy with Limited Light Doses; SPIE Spotlight Series; SPIE: Bellingham, WA, USA, 2018; Volume SL 42. [CrossRef]

21. Demmerle, J.; Innocent, C.; North, A.J.; Ball, G.; Müller, M.; Miron, E.; Matsuda, A.; Dobbie, I.M.; Markaki, Y.; Schermelleh, L. Strategic and practical guidelines for successful structured illumination microscopy. Nat. Protoc. 2017, 12, 988-1010. [CrossRef] [PubMed]

22. Müller, M.; Mönkemöller, V.; Hennig, S.; Hübner, W.; Huser, T. Open-source image reconstruction of super-resolution structured illumination microscopy data in ImageJ. Nat. Commun. 2016, 7, 10980. [CrossRef] [PubMed]

23. Komis, G.; Mistrik, M.; Šamajová, O.; Doskocilová, A.; Ovecka, M.; Illés, P.; Bartek, J.; Šamaj, J. Dynamics and organization of cortical microtubules as revealed by superresolution structured illumination Microscopy. Plant Physiol. 2014, 165, 129-147. [CrossRef] [PubMed]

24. Guo, Y.; Li, D.; Zhang, S.; Yang, Y.; Liu, J.J.; Wang, X.; Liu, C.; Milkie, D.E.; Moore, R.P.; Tulu, U.S.; et al. Visualizing intracellular organelle and cytoskeletal interactions at nanoscale resolution on millisecond timescales. Cell 2018, 175, 1430-1442. [CrossRef] [PubMed]

25. Blum, R.H.; Carter, S.K. Adriamycin. A new anticancer drug with significant clinical activity. Ann. Intern. Med. 1974, 80, 249-259. [CrossRef] [PubMed]

26. Li, Z.X.; Wang, T.T.; Wu, Y.T.; Xu, C.M.; Dong, M.Y.; Sheng, J.Z.; Huang, H.F. Adriamycin induces H2AX phosphorylation in human spermatozoa. Asian J. Androl. 2008, 10, 749-757. [CrossRef]

27. Scaduto, R.C., Jr.; Grotyohann, L.W. Measurement of mitochondrial membrane potential using fluorescent rhodamine derivatives. Biophys. J. 1999, 76, 469-477. [CrossRef]

28. Schneckenburger, H.; Gschwend, M.H.; Strauss, W.S.L.; Sailer, R.; Kron, M.; Steeb, U.; Steiner, R. Energy transfer spectroscopy for measuring mitochondrial metabolism in living cells. Photochem. Photobiol. 1997, 66, 34-41. [CrossRef]

29. Pinotsi, D.; Buell, A.K.; Galvagnion, C.; Dobson, C.M.; Gabriele, S.; Kaminski-Schierle, G.S.; Kaminski, C.F. Direct Observation of Heterogeneous Amyloid Fibril Growth Kinetics via Two-Color Super-Resolution Microscopy. Nano Lett. 2014, 14, 339-345. [CrossRef]

30. Heintzmann, R.; Huser, T. Super-resolution structured illumination microscopy. Chem. Rev. 2017, 117, 13890-13908. [CrossRef] [PubMed]

31. Xue, Y.; So, P.T.C. Three-dimensional super-resolution high-throughput imaging by structured illumination STED microscopy. Opt. Express 2018, 26, 20920-20928. [CrossRef] [PubMed]

32. Lu-Walther, H.-W.; Kielhorn, M.; Förster, R.; Jost, A.; Wicker, K.; Heintzmann, R. fastSIM: A practical implementation of fast structured illumination microscopy. Methods Appl. Fluoresc. 2015, 3, 014001. [CrossRef] [PubMed]

33. Betzig, E. Excitation strategies for optical lattice microscopy. Opt. Express 2005, 13, 3021-3036. [CrossRef] [PubMed]

34. Godin, A.G.; Lounis, B.; Cognet, L. Super-resolution Microscopy Approaches for Live Cell Imaging. Biophys. J. 2014, 107, 1777-1784. [CrossRef]

35. Tønnesen, J.; Krishna Inavalli, V.V.G.; Nägerl, U.V. Super-Resolution Imaging of the Extracellular Space in Living Brain Tissue. Cell 2018, 172, 1108-1121. [CrossRef] [PubMed]

36. Young, L.J.; Ströhl, F.; Kaminski, C.F. A guide to structured illumination TIRF microscopy at high speed with multiple colors. J. Vis. Exp. 2016, 30, 53988. [CrossRef] [PubMed]

37. Förster, T. Zwischenmolekulare Energiewanderung und Fluoreszenz. Ann. Phys. 1948, 437, 55-75. [CrossRef]

(C) 2019 by the authors. Licensee MDPI, Basel, Switzerland. This article is an open access article distributed under the terms and conditions of the Creative Commons Attribution (CC BY) license (http://creativecommons.org/licenses/by/4.0/). 